\title{
General Multivariate Iyengar Type Inequalities
}

\author{
George A. Anastassiou
}

ABSTRACT. Here we give a variety of general multivariate Iyengar type inequalities for not necessarily radial functions defined on the shell and ball. Our approach is based on the polar coordinates in $\mathbb{R}^{N}, N \geq 2$, and the related multivariate polar integration formula. Via this method we transfer well-known univariate Iyengar type inequalities and univariate author's related results into general multivariate Iyengar inequalities.

Keywords: Iyengar inequality, Polar coordinates, Not necessarily radial function, Shell, Ball.

2010 Mathematics Subject Classification: 26D10, 26D15.

\section{BACKGROUND}

In the year 1938, Iyengar [5] proved the following interesting inequality:

Theorem 1.1. Let $f$ be a differentiable function on $[a, b]$ and $\left|f^{\prime}(x)\right| \leq M_{1}$. Then

$$
\left|\int_{a}^{b} f(x) d x-\frac{1}{2}(b-a)(f(a)+f(b))\right| \leq \frac{M_{1}(b-a)^{2}}{4}-\frac{(f(b)-f(a))^{2}}{4 M_{1}} .
$$

In 2001, X.-L. Cheng [4] proved that

Theorem 1.2. Let $f \in C^{2}([a, b])$ and $\left|f^{\prime \prime}(x)\right| \leq M_{2}$. Then

$$
\begin{aligned}
& \left|\int_{a}^{b} f(x) d x-\frac{1}{2}(b-a)(f(a)+f(b))+\frac{1}{8}(b-a)^{2}\left(f^{\prime}(b)-f^{\prime}(a)\right)\right| \\
\leq & \frac{M_{2}}{24}(b-a)^{3}-\frac{(b-a)}{16 M_{2}} \Delta_{1}^{2},
\end{aligned}
$$

where

$$
\Delta_{1}=f^{\prime}(a)-\frac{2(f(b)-f(a))}{(b-a)}+f^{\prime}(b) .
$$

In 1996, Agarwal and Dragomir [1] obtained a generalization of (1.1):

Theorem 1.3. Let $f:[a, b] \rightarrow \mathbb{R}$ be a differentiable function such that for all $x \in[a, b]$ with $M>m$ we have $m \leq f^{\prime}(x) \leq M$. Then

$$
\begin{aligned}
& \left|\int_{a}^{b} f(x) d x-\frac{1}{2}(b-a)(f(a)+f(b))\right| \\
\leq & \frac{(f(b)-f(a)-m(b-a))(M(b-a)-f(b)+f(a))}{2(M-m)} .
\end{aligned}
$$

In [7], Qi proved the following:

Received: 22 January 2019; Accepted: 27 March 2019; Published Online: 29 March 2019

*Corresponding author: G. A. Anastassiou; ganastss@memphis.edu

DOI: $10.33205 / \mathrm{cma} .543560$ 
Theorem 1.4. Let $f:[a, b] \rightarrow \mathbb{R}$ be a twice differentiable function such that for all $x \in[a, b]$ with $M>0$ we have $\left|f^{\prime \prime}(x)\right| \leq M$. Then

$$
\begin{aligned}
& \left|\int_{a}^{b} f(x) d x-\frac{(f(a)+f(b))}{2}(b-a)+\frac{\left(1+Q^{2}\right)}{8}\left(f^{\prime}(b)-f^{\prime}(a)\right)(b-a)^{2}\right| \\
\leq & \frac{M(b-a)^{3}}{24}\left(1-3 Q^{2}\right),
\end{aligned}
$$

where

$$
Q^{2}=\frac{\left(f^{\prime}(a)+f^{\prime}(b)-2\left(\frac{f(b)-f(a)}{b-a}\right)\right)^{2}}{M^{2}(b-a)^{2}-\left(f^{\prime}(b)-f^{\prime}(a)\right)^{2}} .
$$

In 2005, Zheng Liu, [6], proved the following:

Theorem 1.5. Let $f:[a, b] \rightarrow \mathbb{R}$ be a differentiable function such that $f^{\prime}$ is integrable on $[a, b]$ and for all $x \in[a, b]$ with $M>m$ we have

$$
m \leq \frac{f^{\prime}(x)-f^{\prime}(a)}{x-a} \leq M \text { and } m \leq \frac{f^{\prime}(b)-f^{\prime}(x)}{b-x} \leq M .
$$

Then

$$
\begin{gathered}
\mid \int_{a}^{b} f(x) d x-\frac{(f(a)+f(b))}{2}(b-a)+\left(\frac{1+P^{2}}{8}\right)\left(f^{\prime}(b)-f^{\prime}(a)\right)(b-a)^{2} \\
-\left(\frac{1+3 P^{2}}{48}\right)(m+M)(b-a)^{3} \mid \leq \frac{(M-m)(b-a)^{3}}{48}\left(1-3 P^{2}\right),
\end{gathered}
$$

where

$$
P^{2}=\frac{\left(f^{\prime}(a)+f^{\prime}(b)-2\left(\frac{f(b)-f(a)}{b-a}\right)\right)^{2}}{\left(\frac{M-m}{2}\right)^{2}(b-a)^{2}-\left(f^{\prime}(b)-f^{\prime}(a)-\left(\frac{m+M}{2}\right)(b-a)\right)^{2}} .
$$

Next we list some author's related results, (here $L_{\infty}([a, b])$ is the normed space of essentially bounded functions over $[a, b])$ :

Theorem 1.6. ([3]) Let $n \in \mathbb{N}, f \in A C^{n}([a, b])$ (i.e. $f^{(n-1)} \in A C([a, b])$, absolutely continuous functions). We assume that $f^{(n)} \in L_{\infty}([a, b])$. Then

(i)

$$
\begin{aligned}
& \left|\int_{a}^{b} f(x) d x-\sum_{k=0}^{n-1} \frac{1}{(k+1) !}\left[f^{(k)}(a)(t-a)^{k+1}+(-1)^{k} f^{(k)}(b)(b-t)^{k+1}\right]\right| \\
\leq & \frac{\left\|f^{(n)}\right\|_{L_{\infty}([a, b])}}{(n+1) !}\left[(t-a)^{n+1}+(b-t)^{n+1}\right],
\end{aligned}
$$

for all $t \in[a, b]$,

(ii) at $t=\frac{a+b}{2}$, the right hand side of (1.3) is minimized, and we get:

$$
\begin{aligned}
& \left|\int_{a}^{b} f(x) d x-\sum_{k=0}^{n-1} \frac{1}{(k+1) !} \frac{(b-a)^{k+1}}{2^{k+1}}\left[f^{(k)}(a)+(-1)^{k} f^{(k)}(b)\right]\right| \\
\leq & \frac{\left\|f^{(n)}\right\|_{L_{\infty}([a, b])}}{(n+1) !} \frac{(b-a)^{n+1}}{2^{n}},
\end{aligned}
$$


(iii) if $f^{(k)}(a)=f^{(k)}(b)=0$ for all $k=0,1, \ldots, n-1$, then we obtain

$$
\left|\int_{a}^{b} f(x) d x\right| \leq \frac{\left\|f^{(n)}\right\|_{L_{\infty}([a, b])}}{(n+1) !} \frac{(b-a)^{n+1}}{2^{n}}
$$

which is a sharp inequality,

(iv) more generally, for $j=0,1,2, \ldots, N \in \mathbb{N}$, it holds

$$
\begin{aligned}
& \left|\int_{a}^{b} f(x) d x-\sum_{k=0}^{n-1} \frac{1}{(k+1) !}\left(\frac{b-a}{N}\right)^{k+1}\left[j^{k+1} f^{(k)}(a)+(-1)^{k}(N-j)^{k+1} f^{(k)}(b)\right]\right| \\
\leq & \frac{\left\|f^{(n)}\right\|_{L_{\infty}([a, b])}}{(n+1) !}\left(\frac{b-a}{N}\right)^{n+1}\left[j^{n+1}+(N-j)^{n+1}\right],
\end{aligned}
$$

(v) if $f^{(k)}(a)=f^{(k)}(b)=0, k=1, \ldots, n-1$, from (1.4) we get:

$$
\begin{aligned}
& \left|\int_{a}^{b} f(x) d x-\left(\frac{b-a}{N}\right)[j f(a)+(N-j) f(b)]\right| \\
\leq & \frac{\left\|f^{(n)}\right\|_{L_{\infty}([a, b])}}{(n+1) !}\left(\frac{b-a}{N}\right)^{n+1}\left[j^{n+1}+(N-j)^{n+1}\right]
\end{aligned}
$$

for $j=0,1,2, \ldots, N \in \mathbb{N}$,

(vi) when $N=2$ and $j=1$, (1.5) turns to

$$
\left|\int_{a}^{b} f(x) d x-\left(\frac{b-a}{2}\right)(f(a)+f(b))\right| \leq \frac{\left\|f^{(n)}\right\|_{L_{\infty}([a, b])}}{(n+1) !} \frac{(b-a)^{n+1}}{2^{n}}
$$

(vii) when $n=1$ (without any boundary conditions), we get from (1.6) that

$$
\left|\int_{a}^{b} f(x) d x-\left(\frac{b-a}{2}\right)(f(a)+f(b))\right| \leq\left\|f^{\prime}\right\|_{[a, b], \infty} \frac{(b-a)^{2}}{4},
$$

a similar to Iyengar inequality (1.1).

We mention here $L_{1}([a, b])$ is the normed space of integrable functions over $\left.[a, b]\right)$.

Theorem 1.7. ([3]) Let $f \in A C^{n}([a, b]), n \in \mathbb{N}$. Then

(i)

$$
\begin{aligned}
& \quad\left|\int_{a}^{b} f(x) d x-\sum_{k=0}^{n-1} \frac{1}{(k+1) !}\left[f^{(k)}(a)(t-a)^{k+1}+(-1)^{k} f^{(k)}(b)(b-t)^{k+1}\right]\right| \\
& \leq \frac{\left\|f^{(n)}\right\|_{L_{1}([a, b])}}{n !}\left[(t-a)^{n}+(b-t)^{n}\right],
\end{aligned}
$$

for all $t \in[a, b]$,

(ii) at $t=\frac{a+b}{2}$, the right hand side of (1.7) is minimized, and we get:

$$
\begin{aligned}
& \left|\int_{a}^{b} f(x) d x-\sum_{k=0}^{n-1} \frac{1}{(k+1) !} \frac{(b-a)^{k+1}}{2^{k+1}}\left[f^{(k)}(a)+(-1)^{k} f^{(k)}(b)\right]\right| \\
\leq & \frac{\left\|f^{(n)}\right\|_{L_{1}([a, b])}}{n !} \frac{(b-a)^{n}}{2^{n-1}},
\end{aligned}
$$


(iii) if $f^{(k)}(a)=f^{(k)}(b)=0$, for all $k=0,1, \ldots, n-1$, we obtain

$$
\left|\int_{a}^{b} f(x) d x\right| \leq \frac{\left\|f^{(n)}\right\|_{L_{1}([a, b])}}{n !} \frac{(b-a)^{n}}{2^{n-1}}
$$

which is a sharp inequality,

(iv) more generally, for $j=0,1,2, \ldots, N \in \mathbb{N}$, it holds

$$
\begin{aligned}
& \left|\int_{a}^{b} f(x) d x-\sum_{k=0}^{n-1} \frac{1}{(k+1) !}\left(\frac{b-a}{N}\right)^{k+1}\left[j^{k+1} f^{(k)}(a)+(-1)^{k}(N-j)^{k+1} f^{(k)}(b)\right]\right| \\
& \leq \frac{\left\|f^{(n)}\right\|_{L_{1}([a, b])}}{n !}\left(\frac{b-a}{N}\right)^{n}\left[j^{n}+(N-j)^{n}\right]
\end{aligned}
$$

(v) if $f^{(k)}(a)=f^{(k)}(b)=0, k=1, \ldots, n-1$, from (1.8) we get:

$$
\begin{aligned}
& \left|\int_{a}^{b} f(x) d x-\left(\frac{b-a}{N}\right)[j f(a)+(N-j) f(b)]\right| \\
& \leq \frac{\left\|f^{(n)}\right\|_{L_{1}([a, b])}}{n !}\left(\frac{b-a}{N}\right)^{n}\left[j^{n}+(N-j)^{n}\right],
\end{aligned}
$$

for $j=0,1,2, \ldots, N \in \mathbb{N}$,

(vi) when $N=2$ and $j=1$, (1.9) turns to

$$
\left|\int_{a}^{b} f(x) d x-\frac{(b-a)}{2}(f(a)+f(b))\right| \leq \frac{\left\|f^{(n)}\right\|_{L_{1}([a, b])}}{n !} \frac{(b-a)^{n}}{2^{n-1}}
$$

(vii) when $n=1$ (without any boundary conditions), we get from (1.10) that

$$
\left|\int_{a}^{b} f(x) d x-\left(\frac{b-a}{2}\right)(f(a)+f(b))\right| \leq\left\|f^{\prime}\right\|_{L_{1}([a, b])}(b-a) .
$$

We mention here $L_{q}([a, b])$ is the normed space of functions $f$ such that $|f|^{q}$ is integrable over $[a, b])$

Theorem 1.8. ([3]) Let $f \in A C^{n}([a, b]), n \in \mathbb{N} ; p, q>1$ such that $\frac{1}{p}+\frac{1}{q}=1$, and $f^{(n)} \in L_{q}([a, b])$. Then

(i)

$$
\begin{aligned}
& \left|\int_{a}^{b} f(x) d x-\sum_{k=0}^{n-1} \frac{1}{(k+1) !}\left[f^{(k)}(a)(t-a)^{k+1}+(-1)^{k} f^{(k)}(b)(b-t)^{k+1}\right]\right| \\
\leq & \frac{\left\|f^{(n)}\right\|_{L_{q}([a, b])}}{(n-1) !\left(n+\frac{1}{p}\right)(p(n-1)+1)^{\frac{1}{p}}}\left[(t-a)^{n+\frac{1}{p}}+(b-t)^{n+\frac{1}{p}}\right],
\end{aligned}
$$

for all $t \in[a, b]$, 
(ii) at $t=\frac{a+b}{2}$, the right hand side of (1.11) is minimized, and we get:

$$
\begin{aligned}
& \left|\int_{a}^{b} f(x) d x-\sum_{k=0}^{n-1} \frac{1}{(k+1) !} \frac{(b-a)^{k+1}}{2^{k+1}}\left[f^{(k)}(a)+(-1)^{k} f^{(k)}(b)\right]\right| \\
\leq & \frac{\left\|f^{(n)}\right\|_{L_{q}([a, b])}}{(n-1) !\left(n+\frac{1}{p}\right)(p(n-1)+1)^{\frac{1}{p}}} \frac{(b-a)^{n+\frac{1}{p}}}{2^{n-\frac{1}{q}}},
\end{aligned}
$$

(iii) if $f^{(k)}(a)=f^{(k)}(b)=0$, for all $k=0,1, \ldots, n-1$, we obtain

$$
\left|\int_{a}^{b} f(x) d x\right| \leq \frac{\left\|f^{(n)}\right\|_{L_{q}([a, b])}}{(n-1) !\left(n+\frac{1}{p}\right)(p(n-1)+1)^{\frac{1}{p}}} \frac{(b-a)^{n+\frac{1}{p}}}{2^{n-\frac{1}{q}}},
$$

which is a sharp inequality,

(iv) more generally, for $j=0,1,2, \ldots, N \in \mathbb{N}$, it holds

$$
\begin{aligned}
& \left|\int_{a}^{b} f(x) d x-\sum_{k=0}^{n-1} \frac{1}{(k+1) !}\left(\frac{b-a}{N}\right)^{k+1}\left[j^{k+1} f^{(k)}(a)+(-1)^{k}(N-j)^{k+1} f^{(k)}(b)\right]\right| \\
& \leq \frac{\left\|f^{(n)}\right\|_{L_{q}([a, b])}}{(n-1) !\left(n+\frac{1}{p}\right)(p(n-1)+1)^{\frac{1}{p}}}\left(\frac{b-a}{N}\right)^{n+\frac{1}{p}}\left[j^{n+\frac{1}{p}}+(N-j)^{n+\frac{1}{p}}\right],
\end{aligned}
$$

(v) if $f^{(k)}(a)=f^{(k)}(b)=0, k=1, \ldots, n-1$, from (1.12) we get:

$$
\begin{aligned}
& \left|\int_{a}^{b} f(x) d x-\left(\frac{b-a}{N}\right)[j f(a)+(N-j) f(b)]\right| \\
\leq & \frac{\left\|f^{(n)}\right\|_{L_{q}([a, b])}}{(n-1) !\left(n+\frac{1}{p}\right)(p(n-1)+1)^{\frac{1}{p}}}\left(\frac{b-a}{N}\right)^{n+\frac{1}{p}}\left[j^{n+\frac{1}{p}}+(N-j)^{n+\frac{1}{p}}\right],
\end{aligned}
$$

for $j=0,1,2, \ldots, N \in \mathbb{N}$,

(vi) when $N=2$ and $j=1$, (1.13) turns to

$$
\left|\int_{a}^{b} f(x) d x-\frac{(b-a)}{2}(f(a)+f(b))\right| \leq \frac{\left\|f^{(n)}\right\|_{L_{q}([a, b])}}{(n-1) !\left(n+\frac{1}{p}\right)(p(n-1)+1)^{\frac{1}{p}}} \frac{(b-a)^{n+\frac{1}{p}}}{2^{n-\frac{1}{q}}}
$$

(vii) when $n=1$ (without any boundary conditions), we get from (1.14) that

$$
\left|\int_{a}^{b} f(x) d x-\left(\frac{b-a}{2}\right)(f(a)+f(b))\right| \leq \frac{\left\|f^{\prime}\right\|_{L_{q}([a, b])}}{\left(1+\frac{1}{p}\right)} \frac{(b-a)^{1+\frac{1}{p}}}{2^{\frac{1}{p}}} .
$$

We need

Remark 1.1. We define the ball $B(0, R)=\left\{x \in \mathbb{R}^{N}:|x|<R\right\} \subseteq \mathbb{R}^{N}, N \geq 2, R>0$, and the sphere

$$
S^{N-1}:=\left\{x \in \mathbb{R}^{N}:|x|=1\right\},
$$

where $|\cdot|$ is the Euclidean norm. Let $d \omega$ be the element of surface measure on $S^{N-1}$ and

$$
\omega_{N}=\int_{S^{N-1}} d \omega=\frac{2 \pi^{\frac{N}{2}}}{\Gamma\left(\frac{N}{2}\right)}
$$


is the area of $S^{N-1}$.

For $x \in \mathbb{R}^{N}-\{0\}$ we can write uniquely $x=r \omega$, where $r=|x|>0$ and $\omega=\frac{x}{r} \in S^{N-1},|\omega|=1$. Note that $\int_{B(0, R)} d y=\frac{\omega_{N} R^{N}}{N}$ is the Lebesgue measure on the ball, that is the volume of $B(0, R)$, which exactly is $\operatorname{Vol}(B(0, R))=\frac{\pi^{\frac{N}{2}} R^{N}}{\Gamma\left(\frac{N}{2}+1\right)}$.

Following [8, pp. 149-150, exercise 6], and [9, pp. 87-88, Theorem 5.2.2] we can write for $F$ : $\overline{B(0, R)} \rightarrow \mathbb{R}$ a Lebesgue integrable function that

$$
\int_{B(0, R)} F(x) d x=\int_{S^{N-1}}\left(\int_{0}^{R} F(r \omega) r^{N-1} d r\right) d \omega,
$$

and we use this formula a lot.

Typically here the function $f: \overline{B(0, R)} \rightarrow \mathbb{R}$ is not radial. A radial function $f$ is such that there exists a function $g$ with $f(x)=g(r)$, where $r=|x|, r \in[0, R]$, for all $x \in \overline{B(0, R)}$.

We need

Remark 1.2. Let the spherical shell $A:=B\left(0, R_{2}\right)-\overline{B\left(0, R_{1}\right)}, 0<R_{1}<R_{2}, A \subseteq \mathbb{R}^{N}, N \geq 2$, $x \in \bar{A}$. Consider that $f: \bar{A} \rightarrow \mathbb{R}$ is not radial. A radial function $f$ is such that there exists a function $g$ with $f(x)=g(r), r=|x|, r \in\left[R_{1}, R_{2}\right]$, for all $x \in \bar{A}$. Here $x$ can be written uniquely as $x=r \omega$, where $r=|x|>0$ and $\omega=\frac{x}{r} \in S^{N-1},|\omega|=1$, see ([8], p. 149-150 and [2], p. 421), furthermore for $F: \bar{A} \rightarrow \mathbb{R}$ a Lebesgue integrable function we have that

$$
\int_{A} F(x) d x=\int_{S^{N-1}}\left(\int_{R_{1}}^{R_{2}} F(r \omega) r^{N-1} d r\right) d \omega .
$$

Here

$$
\operatorname{Vol}(A)=\frac{\omega_{N}\left(R_{2}^{N}-R_{1}^{N}\right)}{N}=\frac{\pi^{\frac{N}{2}}\left(R_{2}^{N}-R_{1}^{N}\right)}{\Gamma\left(\frac{N}{2}+1\right)} .
$$

In this article we derive general multivariate Iyengar type inequalities on the shell and ball of $\mathbb{R}^{N}, N \geq 2$, for not necessarily radial functions. Our results are based on Theorems 1.1-1.8.

\section{MAin Results}

We present the following non-radial multivariate Iyengar type inequalities:

We start with

Theorem 2.9. Let the spherical shell $A:=B\left(0, R_{2}\right)-\overline{B\left(0, R_{1}\right)}, 0<R_{1}<R_{2}, A \subseteq \mathbb{R}^{N}, N \geq 2$. Consider $f: \bar{A} \rightarrow \mathbb{R}$ that is not necessarily radial, and that $f \in C^{1}(\bar{A})$. Assume that $\left|\frac{\partial f(s \omega)}{\partial s}\right| \leq M_{1}$, for all $s \in\left[R_{1}, R_{2}\right]$, and for all $\omega \in S^{N-1}$, where $M_{1}>0$.

Then

$$
\begin{aligned}
& \left|\int_{A} f(y) d y-\frac{\left(R_{2}-R_{1}\right)}{2}\left(R_{1}^{N-1} \int_{S^{N-1}} f\left(R_{1} \omega\right) d \omega+R_{2}^{N-1} \int_{S^{N-1}} f\left(R_{2} \omega\right) d \omega\right)\right| \\
\leq & \frac{M_{1} \pi^{\frac{N}{2}}\left(R_{2}-R_{1}\right)^{2}}{2 \Gamma\left(\frac{N}{2}\right)}-\frac{\int_{S^{N-1}}\left(f\left(R_{2} \omega\right) R_{2}^{N-1}-f\left(R_{1} \omega\right) R_{1}^{N-1}\right)^{2} d \omega}{4 M_{1}} .
\end{aligned}
$$


Proof. Here $f(s \omega) s^{N-1} \in C^{1}\left(\left[R_{1}, R_{2}\right]\right), N \geq 2$, for all $\omega \in S^{N-1}$. By (1.1) we get

$$
\begin{aligned}
& \quad\left|\int_{R_{1}}^{R_{2}} f(s \omega) s^{N-1} d s-\frac{1}{2}\left(R_{2}-R_{1}\right)\left(f\left(R_{1} \omega\right) R_{1}^{N-1}+f\left(R_{2} \omega\right) R_{2}^{N-1}\right)\right| \\
& \leq \frac{M_{1}\left(R_{2}-R_{1}\right)^{2}}{4}-\frac{\left(f\left(R_{2} \omega\right) R_{2}^{N-1}-f\left(R_{1} \omega\right) R_{1}^{N-1}\right)^{2}}{4 M_{1}}=: \lambda_{1}(\omega),
\end{aligned}
$$

for all $\omega \in S^{N-1}$.

Equivalently, we have

$$
-\lambda_{1}(\omega) \leq \int_{R_{1}}^{R_{2}} f(s \omega) s^{N-1} d s-\frac{1}{2}\left(R_{2}-R_{1}\right)\left(f\left(R_{1} \omega\right) R_{1}^{N-1}+f\left(R_{2} \omega\right) R_{2}^{N-1}\right) \leq \lambda_{1}(\omega),
$$

for all $\omega \in S^{N-1}$.

Hence it holds

$$
\begin{aligned}
& -\int_{S^{N-1}} \lambda_{1}(\omega) d \omega \leq \int_{S^{N-1}}\left(\int_{R_{1}}^{R_{2}} f(s \omega) s^{N-1} d s\right) d \omega \\
& -\frac{1}{2}\left(R_{2}-R_{1}\right)\left(R_{1}^{N-1} \int_{S^{N-1}} f\left(R_{1} \omega\right) d \omega+R_{2}^{N-1} \int_{S^{N-1}} f\left(R_{2} \omega\right) d \omega\right) \\
& \leq \int_{S^{N-1}} \lambda_{1}(\omega) d \omega .
\end{aligned}
$$

That is (by (1.16))

$$
\begin{aligned}
& -\left[\frac{\pi^{\frac{N}{2}} M_{1}\left(R_{2}-R_{1}\right)^{2}}{2 \Gamma\left(\frac{N}{2}\right)}-\frac{\int_{S^{N-1}}\left(f\left(R_{2} \omega\right) R_{2}^{N-1}-f\left(R_{1} \omega\right) R_{1}^{N-1}\right)^{2} d \omega}{4 M_{1}}\right] \\
& \leq \int_{A} f(y) d y-\frac{\left(R_{2}-R_{1}\right)}{2}\left(R_{1}^{N-1} \int_{S^{N-1}} f\left(R_{1} \omega\right) d \omega+R_{2}^{N-1} \int_{S^{N-1}} f\left(R_{2} \omega\right) d \omega\right) \\
& \leq \frac{\pi^{\frac{N}{2}} M_{1}\left(R_{2}-R_{1}\right)^{2}}{2 \Gamma\left(\frac{N}{2}\right)}-\frac{\int_{S^{N-1}}\left(f\left(R_{2} \omega\right) R_{2}^{N-1}-f\left(R_{1} \omega\right) R_{1}^{N-1}\right)^{2} d \omega}{4 M_{1}},
\end{aligned}
$$

proving the claim.

We continue with

Theorem 2.10. Let the spherical shell $A:=B\left(0, R_{2}\right)-\overline{B\left(0, R_{1}\right)}, 0<R_{1}<R_{2}, A \subseteq \mathbb{R}^{N}, N \geq 2$. Consider $f: \bar{A} \rightarrow \mathbb{R}$ that is not necessarily radial, and that $f \in C^{2}(\bar{A})$. Assume that $\left|\frac{\partial^{2} f(s \omega)}{\partial s^{2}}\right| \leq M_{2}$, for all $s \in\left[R_{1}, R_{2}\right]$, and for all $\omega \in S^{N-1}$, where $M_{2}>0$.

Set

$$
\begin{aligned}
& \Delta_{1}(\omega):=\left(f(s \omega) s^{N-1}\right)^{\prime}\left(R_{1}\right)-\frac{2\left(f\left(R_{2} \omega\right) R_{2}^{N-1}-f\left(R_{1} \omega\right) R_{1}^{N-1}\right)}{R_{2}-R_{1}} \\
&+\quad\left(f(s \omega) s^{N-1}\right)^{\prime}\left(R_{2}\right), \quad \forall \omega \in S^{N-1} .
\end{aligned}
$$


Then

$$
\begin{aligned}
& \mid \int_{A} f(y) d y-\frac{\left(R_{2}-R_{1}\right)}{2}\left(R_{1}^{N-1} \int_{S^{N-1}} f\left(R_{1} \omega\right) d \omega+R_{2}^{N-1} \int_{S^{N-1}} f\left(R_{2} \omega\right) d \omega\right) \\
+ & \frac{\left(R_{2}-R_{1}\right)^{2}}{8}\left[\int_{S^{N-1}}\left(f(s \omega) s^{N-1}\right)^{\prime}\left(R_{2}\right) d \omega-\int_{S^{N-1}}\left(f(s \omega) s^{N-1}\right)^{\prime}\left(R_{1}\right) d \omega\right] \mid \\
\leq & \frac{\pi^{\frac{N}{2}}}{\Gamma\left(\frac{N}{2}\right)} \frac{M_{2}}{12}\left(R_{2}-R_{1}\right)^{3}-\frac{\left(R_{2}-R_{1}\right)}{16 M_{2}} \int_{S^{N-1}} \Delta_{1}^{2}(\omega) d \omega .
\end{aligned}
$$

Proof. Here $f(s \omega) s^{N-1} \in C^{2}\left(\left[R_{1}, R_{2}\right]\right), N \geq 2$, for all $\omega \in S^{N-1}$. By (1.2) we get

$$
\begin{aligned}
& \mid \int_{R_{1}}^{R_{2}} f(s \omega) s^{N-1} d s-\frac{1}{2}\left(R_{2}-R_{1}\right)\left(f\left(R_{1} \omega\right) R_{1}^{N-1}+f\left(R_{2} \omega\right) R_{2}^{N-1}\right) \\
+ & \frac{1}{8}\left(R_{2}-R_{1}\right)^{2}\left(\left(f(s \omega) s^{N-1}\right)^{\prime}\left(R_{2}\right)-\left(f(s \omega) s^{N-1}\right)^{\prime}\left(R_{1}\right)\right) \mid \\
\leq & \frac{M_{2}}{24}\left(R_{2}-R_{1}\right)^{3}-\frac{\left(R_{2}-R_{1}\right)}{16 M_{2}} \Delta_{1}^{2}(\omega)=: \lambda_{2}(\omega),
\end{aligned}
$$

for all $\omega \in S^{N-1}$.

Equivalently, we have

$$
\begin{aligned}
& -\quad \lambda_{2}(\omega) \leq \int_{R_{1}}^{R_{2}} f(s \omega) s^{N-1} d s-\frac{\left(R_{2}-R_{1}\right)}{2}\left(f\left(R_{1} \omega\right) R_{1}^{N-1}+f\left(R_{2} \omega\right) R_{2}^{N-1}\right) \\
& +\frac{1}{8}\left(R_{2}-R_{1}\right)^{2}\left(\left(f(s \omega) s^{N-1}\right)^{\prime}\left(R_{2}\right)-\left(f(s \omega) s^{N-1}\right)^{\prime}\left(R_{1}\right)\right) \leq \lambda_{2}(\omega),
\end{aligned}
$$

for all $\omega \in S^{N-1}$.

Hence it holds

$$
\begin{aligned}
& -\int_{S^{N-1}} \lambda_{2}(\omega) d \omega \leq \int_{S^{N-1}}\left(\int_{R_{1}}^{R_{2}} f(s \omega) s^{N-1} d s\right) d \omega \\
& -\frac{\left(R_{2}-R_{1}\right)}{2}\left(R_{1}^{N-1} \int_{S^{N-1}} f\left(R_{1} \omega\right) d \omega+R_{2}^{N-1} \int_{S^{N-1}} f\left(R_{2} \omega\right) d \omega\right) \\
& +\frac{\left(R_{2}-R_{1}\right)^{2}}{8}\left[\int_{S^{N-1}}\left(f(s \omega) s^{N-1}\right)^{\prime}\left(R_{2}\right) d \omega-\int_{S^{N-1}}\left(f(s \omega) s^{N-1}\right)^{\prime}\left(R_{1}\right) d \omega\right] \\
& \leq \int_{S^{N-1}} \lambda_{2}(\omega) d \omega .
\end{aligned}
$$

That is (by (1.16))

$$
\begin{aligned}
& -\left[\frac{\pi^{\frac{N}{2}}}{\Gamma\left(\frac{N}{2}\right)} \frac{M_{2}}{12}\left(R_{2}-R_{1}\right)^{3}-\frac{\left(R_{2}-R_{1}\right)}{16 M_{2}} \int_{S^{N-1}} \Delta_{1}^{2}(\omega) d \omega\right] \\
& \leq \int_{A} f(y) d y-\frac{\left(R_{2}-R_{1}\right)}{2}\left(R_{1}^{N-1} \int_{S^{N-1}} f\left(R_{1} \omega\right) d \omega+R_{2}^{N-1} \int_{S^{N-1}} f\left(R_{2} \omega\right) d \omega\right) \\
& +\frac{\left(R_{2}-R_{1}\right)^{2}}{8}\left[\int_{S^{N-1}}\left(f(s \omega) s^{N-1}\right)^{\prime}\left(R_{2}\right) d \omega-\int_{S^{N-1}}\left(f(s \omega) s^{N-1}\right)^{\prime}\left(R_{1}\right) d \omega\right] \\
& \leq \frac{\pi^{\frac{N}{2}}}{\Gamma\left(\frac{N}{2}\right)} \frac{M_{2}}{12}\left(R_{2}-R_{1}\right)^{3}-\frac{\left(R_{2}-R_{1}\right)}{16 M_{2}} \int_{S^{N-1}} \Delta_{1}^{2}(\omega) d \omega,
\end{aligned}
$$


proving the claim.

We give

Theorem 2.11. Let the spherical shell $A:=B\left(0, R_{2}\right)-\overline{B\left(0, R_{1}\right)}, 0<R_{1}<R_{2}, A \subseteq \mathbb{R}^{N}, N \geq 2$. Consider $f: \bar{A} \rightarrow \mathbb{R}$ that is not necessarily radial, and that $f \in C^{1}(\bar{A})$. Let $M>m$ and assume that $m \leq \frac{\partial f(s \omega)}{\partial s} \leq M$, for all $s \in\left[R_{1}, R_{2}\right]$, and for all $\omega \in S^{N-1}$.

Then

$$
\begin{aligned}
& \left|\int_{A} f(y) d y-\frac{\left(R_{2}-R_{1}\right)}{2}\left(R_{1}^{N-1} \int_{S^{N-1}} f\left(R_{1} \omega\right) d \omega+R_{2}^{N-1} \int_{S^{N-1}} f\left(R_{2} \omega\right) d \omega\right)\right| \\
\leq & \frac{1}{2(M-m)} \int_{S^{N-1}}\left[\left(f\left(R_{2} \omega\right) R_{2}^{N-1}-f\left(R_{1} \omega\right) R_{1}^{N-1}-m\left(R_{2}-R_{1}\right)\right)\right. \\
\times \quad & \left.\left(M\left(R_{2}-R_{1}\right)-f\left(R_{2} \omega\right) R_{2}^{N-1}+f\left(R_{1} \omega\right) R_{1}^{N-1}\right)\right] d \omega .
\end{aligned}
$$

Proof. Similar to the proof of Theorem 2.9 by using Theorem 1.3 and (1.16).

We give

Theorem 2.12. Let the spherical shell $A:=B\left(0, R_{2}\right)-\overline{B\left(0, R_{1}\right)}, 0<R_{1}<R_{2}, A \subseteq \mathbb{R}^{N}, N \geq 2$. Consider $f: \bar{A} \rightarrow \mathbb{R}$ that is not necessarily radial, and that $f \in C^{2}(\bar{A})$. Assume that $\left|\frac{\partial^{2} f(s \omega)}{\partial s^{2}}\right| \leq M_{3}$, for all $s \in\left[R_{1}, R_{2}\right]$, and for all $\omega \in S^{N-1}$, where $M_{3}>0$.

Set

$$
\begin{aligned}
& Q_{1}^{2}(\omega) \\
& :=\frac{\left[\left(f(s \omega) s^{N-1}\right)^{\prime}\left(R_{1}\right)+\left(f(s \omega) s^{N-1}\right)^{\prime}\left(R_{2}\right)-2\left(\frac{f\left(R_{2} \omega\right) R_{2}^{N-1}-f\left(R_{1} \omega\right) R_{1}^{N-1}}{R_{2}-R_{1}}\right)\right]^{2}}{\left[M_{3}^{2}\left(R_{2}-R_{1}\right)^{2}-\left(\left(f(s \omega) s^{N-1}\right)^{\prime}\left(R_{2}\right)-\left(f(s \omega) s^{N-1}\right)^{\prime}\left(R_{1}\right)\right)^{2}\right]},
\end{aligned}
$$

for all $\omega \in S^{N-1}$.

Then

$$
\begin{aligned}
& \mid \int_{A} f(y) d y-\frac{\left(R_{2}-R_{1}\right)}{2}\left(R_{1}^{N-1} \int_{S^{N-1}} f\left(R_{1} \omega\right) d \omega+R_{2}^{N-1} \int_{S^{N-1}} f\left(R_{2} \omega\right) d \omega\right) \\
+ & \frac{\left(R_{2}-R_{1}\right)^{2}}{8} \int_{S^{N-1}}\left(1+Q_{1}^{2}(\omega)\right)\left(\left(f(s \omega) s^{N-1}\right)^{\prime}\left(R_{2}\right)-\left(f(s \omega) s^{N-1}\right)^{\prime}\left(R_{1}\right)\right) d \omega \mid \\
\leq & \frac{M_{3}\left(R_{2}-R_{1}\right)^{3}}{24} \int_{S^{N-1}}\left(1-3 Q_{1}^{2}(\omega)\right) d \omega .
\end{aligned}
$$

Proof. Similar to the proof of Theorem 2.10 by using Theorem 1.4 and (1.16).

We continue with

Theorem 2.13. Here all as in Theorem 2.9, and let $M_{1}>m_{1}$. Assume that

$$
m_{1} \leq \frac{\left(f(s \omega) s^{N-1}\right)^{\prime}(x)-\left(f(s \omega) s^{N-1}\right)^{\prime}\left(R_{1}\right)}{x-R_{1}} \leq M_{1},
$$

and

$$
m_{1} \leq \frac{\left(f(s \omega) s^{N-1}\right)^{\prime}\left(R_{2}\right)-\left(f(s \omega) s^{N-1}\right)^{\prime}(x)}{R_{2}-x} \leq M_{1},
$$

for all $x \in\left[R_{1}, R_{2}\right]$, for all $\omega \in S^{N-1}$. 
Set

$$
\begin{aligned}
& P_{1}^{2}(\omega) \\
& =\frac{\left[\left(f(s \omega) s^{N-1}\right)^{\prime}\left(R_{1}\right)+\left(f(s \omega) s^{N-1}\right)^{\prime}\left(R_{2}\right)-2\left(\frac{f\left(R_{2} \omega\right) R_{2}^{N-1}-f\left(R_{1} \omega\right) R_{1}^{N-1}}{R_{2}-R_{1}}\right)\right]^{2}}{\left(\frac{M_{1}-m_{1}}{2}\right)^{2}\left(R_{2}-R_{1}\right)^{2}-\left[\left(f(s \omega) s^{N-1}\right)^{\prime}\left(R_{2}\right)-\left(f(s \omega) s^{N-1}\right)^{\prime}\left(R_{1}\right)-\left(\frac{m_{1}+M_{1}}{2}\right)\left(R_{2}-R_{1}\right)\right]^{2}},
\end{aligned}
$$

for all $\omega \in S^{N-1}$.

Then

$$
\begin{aligned}
& \mid \int_{A} f(y) d y-\left(\frac{R_{2}-R_{1}}{2}\right)\left(R_{1}^{N-1} \int_{S^{N-1}} f\left(R_{1} \omega\right) d \omega+R_{2}^{N-1} \int_{S^{N-1}} f\left(R_{2} \omega\right) d \omega\right) \\
+ & \frac{\left(R_{2}-R_{1}\right)^{2}}{8} \int_{S^{N-1}}\left(1+P_{1}^{2}(\omega)\right)\left(\left(f(s \omega) s^{N-1}\right)^{\prime}\left(R_{2}\right)-\left(f(s \omega) s^{N-1}\right)^{\prime}\left(R_{1}\right)\right) d \omega \\
- & \frac{\left(R_{2}-R_{1}\right)^{3}}{48}\left(m_{1}+M_{1}\right) \int_{S^{N-1}}\left(1+3 P_{1}^{2}(\omega)\right) d \omega \mid \\
\leq & \frac{\left(M_{1}-m_{1}\right)\left(R_{2}-R_{1}\right)^{3}}{48} \int_{S^{N-1}}\left(1-3 P_{1}^{2}(\omega)\right) d \omega .
\end{aligned}
$$

Proof. Similar to the proof of Theorem 2.10 by using Theorem 1.5 and (1.16).

\section{We present}

Theorem 2.14. Consider $f: \bar{A} \rightarrow \mathbb{R}$ be Lebesgue integrable, which is not necessarily radial. Assume that $f(s \omega) s^{N-1} \in A C^{n}\left(\left[R_{1}, R_{2}\right]\right)$ (i.e. $\left(f(s \omega) s^{N-1}\right)^{(n-1)} \in A C\left(\left[R_{1}, R_{2}\right]\right)$ absolutely continuous functions), for all $\omega \in S^{N-1}, N \geq 2$. We assume that $\left(f(s \omega) s^{N-1}\right)^{(n)} \in L_{\infty}\left(\left[R_{1}, R_{2}\right]\right)$, for all $\omega \in S^{N-1}$. There exists $K_{1}>0$ such that $\left\|\left(f(s \omega) s^{N-1}\right)^{(n)}\right\|_{L_{\infty}\left(\left[R_{1}, R_{2}\right]\right)} \leq K_{1}$, where $s \in\left[R_{1}, R_{2}\right]$, for all $\omega \in S^{N-1}$.

Then

(i)

$$
\begin{aligned}
& \mid \int_{A} f(y) d y-\sum_{k=0}^{n-1} \frac{1}{(k+1) !}\left[\left(\int_{S^{N-1}}\left(f(s \omega) s^{N-1}\right)^{(k)}\left(R_{1}\right) d \omega\right)\left(t-R_{1}\right)^{k+1}\right. \\
+ & \left.(-1)^{k}\left(\int_{S^{N-1}}\left(f(s \omega) s^{N-1}\right)^{(k)}\left(R_{2}\right) d \omega\right)\left(R_{2}-t\right)^{k+1}\right] \mid \\
\leq & \frac{2 \pi^{\frac{N}{2}}}{\Gamma\left(\frac{N}{2}\right)} \frac{K_{1}}{(n+1) !}\left[\left(t-R_{1}\right)^{n+1}+\left(R_{2}-t\right)^{n+1}\right],
\end{aligned}
$$

for all $t \in\left[R_{1}, R_{2}\right]$,

(ii) at $t=\frac{R_{1}+R_{2}}{2}$, the right hand side of (2.17) is minimized, and we get:

$$
\begin{aligned}
& \mid \int_{A} f(y) d y-\sum_{k=0}^{n-1} \frac{1}{(k+1) !} \frac{\left(R_{2}-R_{1}\right)^{k+1}}{2^{k+1}}\left[\int_{S^{N-1}}\left(f(s \omega) s^{N-1}\right)^{(k)}\left(R_{1}\right) d \omega\right. \\
+ & \left.(-1)^{k} \int_{S^{N-1}}\left(f(s \omega) s^{N-1}\right)^{(k)}\left(R_{2}\right) d \omega\right] \mid \\
\leq & \frac{\pi^{\frac{N}{2}}}{\Gamma\left(\frac{N}{2}\right)} \frac{K_{1}}{(n+1) !} \frac{\left(R_{2}-R_{1}\right)^{n+1}}{2^{n-1}},
\end{aligned}
$$


(iii) if $\left(f(s \omega) s^{N-1}\right)^{(k)}\left(R_{1}\right)=\left(f(s \omega) s^{N-1}\right)^{(k)}\left(R_{2}\right)=0$, for all $\omega \in S^{N-1}$, (i.e. $\frac{\partial^{k}\left(f(s \omega) s^{N-1}\right)}{\partial s^{k}}$ vanish on $\partial B\left(0, R_{1}\right)$ and $\left.\partial B\left(0, R_{2}\right)\right)$ for all $k=0,1, \ldots, n-1$, we obtain

$$
\left|\int_{A} f(y) d y\right| \leq \frac{\pi^{\frac{N}{2}}}{\Gamma\left(\frac{N}{2}\right)} \frac{K_{1}}{(n+1) !} \frac{\left(R_{2}-R_{1}\right)^{n+1}}{2^{n-1}}
$$

which is a sharp inequality,

(iv) more generally, for $j=0,1,2, \ldots, \bar{N} \in \mathbb{N}$, it holds

$$
\begin{aligned}
& \mid \int_{A} f(y) d y-\sum_{k=0}^{n-1} \frac{1}{(k+1) !}\left(\frac{R_{2}-R_{1}}{\bar{N}}\right)^{k+1}\left[j^{k+1}\left(\int_{S^{N-1}}\left(f(s \omega) s^{N-1}\right)^{(k)}\left(R_{1}\right) d \omega\right)\right. \\
& \left.+\quad(-1)^{k}(\bar{N}-j)^{k+1}\left(\int_{S^{N-1}}\left(f(s \omega) s^{N-1}\right)^{(k)}\left(R_{2}\right) d \omega\right)\right] \mid \\
& \leq \frac{2 \pi^{\frac{N}{2}}}{\Gamma\left(\frac{N}{2}\right)} \frac{K_{1}}{(n+1) !}\left(\frac{R_{2}-R_{1}}{\bar{N}}\right)^{n+1}\left[j^{n+1}+(\bar{N}-j)^{n+1}\right],
\end{aligned}
$$

(v) if $\left(f(s \omega) s^{N-1}\right)^{(k)}\left(R_{1}\right)=\left(f(s \omega) s^{N-1}\right)^{(k)}\left(R_{2}\right)=0$, for all $\omega \in S^{N-1}$, (i.e. $\frac{\partial^{k}\left(f(s \omega) s^{N-1}\right)}{\partial s^{k}}$ vanish on $\partial B\left(0, R_{1}\right)$ and $\left.\partial B\left(0, R_{2}\right)\right)$ for $k=1, \ldots, n-1$, from (2.18) we get:

$$
\begin{aligned}
& \mid \int_{A} f(y) d y-\left(\frac{R_{2}-R_{1}}{\bar{N}}\right)\left[j R_{1}^{N-1}\left(\int_{S^{N-1}} f\left(R_{1} \omega\right) d \omega\right)\right. \\
+ & \left.(\bar{N}-j) R_{2}^{N-1}\left(\int_{S^{N-1}} f\left(R_{2} \omega\right) d \omega\right)\right] \mid \leq \frac{2 \pi^{\frac{N}{2}}}{\Gamma\left(\frac{N}{2}\right)} \cdot \\
\times & \frac{K_{1}}{(n+1) !}\left(\frac{R_{2}-R_{1}}{\bar{N}}\right)^{n+1}\left[j^{n+1}+(\bar{N}-j)^{n+1}\right],
\end{aligned}
$$

for $j=0,1,2, \ldots, \bar{N} \in \mathbb{N}$,

(vi) when $\bar{N}=2$ and $j=1$, (2.19) turns to

$$
\begin{aligned}
& \left|\int_{A} f(y) d y-\left(\frac{R_{2}-R_{1}}{2}\right)\left(R_{1}^{N-1} \int_{S^{N-1}} f\left(R_{1} \omega\right) d \omega+R_{2}^{N-1} \int_{S^{N-1}} f\left(R_{2} \omega\right) d \omega\right)\right| \\
\leq & \frac{\pi^{\frac{N}{2}}}{\Gamma\left(\frac{N}{2}\right)} \frac{K_{1}}{(n+1) !} \frac{\left(R_{2}-R_{1}\right)^{n+1}}{2^{n-1}},
\end{aligned}
$$

(vii) when $n=1$ (without any boundary conditions), we get from (2.20) that

$$
\begin{aligned}
& \left|\int_{A} f(y) d y-\left(\frac{R_{2}-R_{1}}{2}\right)\left(R_{1}^{N-1} \int_{S^{N-1}} f\left(R_{1} \omega\right) d \omega+R_{2}^{N-1} \int_{S^{N-1}} f\left(R_{2} \omega\right) d \omega\right)\right| \\
\leq & \frac{\pi^{\frac{N}{2}} K_{1}}{2 \Gamma\left(\frac{N}{2}\right)}\left(R_{2}-R_{1}\right)^{2} .
\end{aligned}
$$

Proof. Similar to the proof of Theorem 2.9. We apply Theorem 1.6 along with (1.16).

We continue with

Theorem 2.15. Consider $f: \bar{A} \rightarrow \mathbb{R}$ be Lebesgue integrable, which is not necessarily radial. Assume that $f(s \omega) s^{N-1} \in A C^{n}\left(\left[R_{1}, R_{2}\right]\right)$ i.e. $\left(f(s \omega) s^{N-1}\right)^{(n-1)} \in A C\left(\left[R_{1}, R_{2}\right]\right)$ absolutely continuous functions), for all $\omega \in S^{N-1}, N \geq 2$. Here there exists $K_{2}>0$ such that $\left\|\left(f(s \omega) s^{N-1}\right)^{(n)}\right\|_{L_{1}\left(\left[R_{1}, R_{2}\right]\right)} \leq K_{2}$, where $s \in\left[R_{1}, R_{2}\right]$, for all $\omega \in S^{N-1}$.

Then 
(i)

(2.21)

$$
\begin{aligned}
& \mid \int_{A} f(y) d y-\sum_{k=0}^{n-1} \frac{1}{(k+1) !}\left[\left(\int_{S^{N-1}}\left(f(s \omega) s^{N-1}\right)^{(k)}\left(R_{1}\right) d \omega\right)\left(t-R_{1}\right)^{k+1}\right. \\
+ & \left.(-1)^{k}\left(\int_{S^{N-1}}\left(f(s \omega) s^{N-1}\right)^{(k)}\left(R_{2}\right) d \omega\right)\left(R_{2}-t\right)^{k+1}\right] \mid \\
\leq & \frac{2 \pi^{\frac{N}{2}}}{\Gamma\left(\frac{N}{2}\right)} \frac{K_{2}}{n !}\left[\left(t-R_{1}\right)^{n}+\left(R_{2}-t\right)^{n}\right],
\end{aligned}
$$

for all $t \in\left[R_{1}, R_{2}\right]$,

(ii) at $t=\frac{R_{1}+R_{2}}{2}$, the right hand side of (2.21) is minimized, and we get:

$$
\begin{aligned}
& \mid \int_{A} f(y) d y-\sum_{k=0}^{n-1} \frac{1}{(k+1) !} \frac{\left(R_{2}-R_{1}\right)^{k+1}}{2^{k+1}}\left[\int_{S^{N-1}}\left(f(s \omega) s^{N-1}\right)^{(k)}\left(R_{1}\right) d \omega\right. \\
+ & \left.(-1)^{k} \int_{S^{N-1}}\left(f(s \omega) s^{N-1}\right)^{(k)}\left(R_{2}\right) d \omega\right] \mid \\
\leq & \frac{\pi^{\frac{N}{2}}}{\Gamma\left(\frac{N}{2}\right)} \frac{K_{2}}{n !} \frac{\left(R_{2}-R_{1}\right)^{n}}{2^{n-2}},
\end{aligned}
$$

(iii) if $\left(f(s \omega) s^{N-1}\right)^{(k)}\left(R_{1}\right)=\left(f(s \omega) s^{N-1}\right)^{(k)}\left(R_{2}\right)=0$, for all $\omega \in S^{N-1}$, (i.e. $\frac{\partial^{k}\left(f(s \omega) s^{N-1}\right)}{\partial s^{k}}$ vanish on $\partial B\left(0, R_{1}\right)$ and $\left.\partial B\left(0, R_{2}\right)\right)$ for all $k=0,1, \ldots, n-1$, we obtain

$$
\left|\int_{A} f(y) d y\right| \leq \frac{\pi^{\frac{N}{2}}}{\Gamma\left(\frac{N}{2}\right)} \frac{K_{2}}{n !} \frac{\left(R_{2}-R_{1}\right)^{n}}{2^{n-2}},
$$

which is a sharp inequality,

(iv) more generally, for $j=0,1,2, \ldots, \bar{N} \in \mathbb{N}$, it holds

$$
\begin{aligned}
& \mid \int_{A} f(y) d y-\sum_{k=0}^{n-1} \frac{1}{(k+1) !}\left(\frac{R_{2}-R_{1}}{\bar{N}}\right)^{k+1}\left[j^{k+1}\left(\int_{S^{N-1}}\left(f(s \omega) s^{N-1}\right)^{(k)}\left(R_{1}\right) d \omega\right)\right. \\
& \left.+\quad(-1)^{k}(\bar{N}-j)^{k+1}\left(\int_{S^{N-1}}\left(f(s \omega) s^{N-1}\right)^{(k)}\left(R_{2}\right) d \omega\right)\right] \mid \\
& \leq \frac{2 \pi^{\frac{N}{2}}}{\Gamma\left(\frac{N}{2}\right)} \frac{K_{2}}{n !}\left(\frac{R_{2}-R_{1}}{\bar{N}}\right)^{n}\left[j^{n}+(\bar{N}-j)^{n}\right],
\end{aligned}
$$

(v) if $\left(f(s \omega) s^{N-1}\right)^{(k)}\left(R_{1}\right)=\left(f(s \omega) s^{N-1}\right)^{(k)}\left(R_{2}\right)=0$, for all $\omega \in S^{N-1}$, (i.e. $\frac{\partial^{k}\left(f(s \omega) s^{N-1}\right)}{\partial s^{k}}$ vanish on $\partial B\left(0, R_{1}\right)$ and $\left.\partial B\left(0, R_{2}\right)\right)$ for $k=1, \ldots, n-1$, from (2.22) we get:

$$
\begin{aligned}
& \mid \int_{A} f(y) d y-\left(\frac{R_{2}-R_{1}}{\bar{N}}\right)\left[j R_{1}^{N-1}\left(\int_{S^{N-1}} f\left(R_{1} \omega\right) d \omega\right)\right. \\
& \left.+\quad(\bar{N}-j) R_{2}^{N-1}\left(\int_{S^{N-1}} f\left(R_{2} \omega\right) d \omega\right)\right] \mid \leq \frac{2 \pi^{\frac{N}{2}}}{\Gamma\left(\frac{N}{2}\right)} \\
& \times \quad \frac{K_{2}}{n !}\left(\frac{R_{2}-R_{1}}{\bar{N}}\right)^{n}\left[j^{n}+(\bar{N}-j)^{n}\right],
\end{aligned}
$$

for $j=0,1,2, \ldots, \bar{N} \in \mathbb{N}$, 
(vi) when $\bar{N}=2$ and $j=1$, (2.23) turns to

$$
\begin{aligned}
& \left|\int_{A} f(y) d y-\left(\frac{R_{2}-R_{1}}{2}\right)\left(R_{1}^{N-1} \int_{S^{N-1}} f\left(R_{1} \omega\right) d \omega+R_{2}^{N-1} \int_{S^{N-1}} f\left(R_{2} \omega\right) d \omega\right)\right| \\
\leq & \frac{\pi^{\frac{N}{2}}}{\Gamma\left(\frac{N}{2}\right)} \frac{K_{2}}{n !} \frac{\left(R_{2}-R_{1}\right)^{n}}{2^{n-2}}
\end{aligned}
$$

(vii) when $n=1$ (without any boundary conditions), we get from (2.24) that

$$
\begin{aligned}
& \left|\int_{A} f(y) d y-\left(\frac{R_{2}-R_{1}}{2}\right)\left(R_{1}^{N-1} \int_{S^{N-1}} f\left(R_{1} \omega\right) d \omega+R_{2}^{N-1} \int_{S^{N-1}} f\left(R_{2} \omega\right) d \omega\right)\right| \\
\leq & \frac{2 \pi^{\frac{N}{2}} K_{2}}{\Gamma\left(\frac{N}{2}\right)}\left(R_{2}-R_{1}\right) .
\end{aligned}
$$

Proof. Similar to the proof of Theorem 2.9. We apply Theorem 1.7 along with (1.16).

We continue with

Theorem 2.16. Let $p, q>1: \frac{1}{p}+\frac{1}{q}>1$. Consider $f: \bar{A} \rightarrow \mathbb{R}$ be Lebesgue integrable, which is not necessarily radial. Assume that $f(s \omega) s^{N-1} \in A C^{n}\left(\left[R_{1}, R_{2}\right]\right)$ (i.e. $\left(f(s \omega) s^{N-1}\right)^{(n-1)} \in A C\left(\left[R_{1}, R_{2}\right]\right)$ absolutely continuous functions), for all $\omega \in S^{N-1}, N \geq 2$. We assume that $\left(f(s \omega) s^{N-1}\right)^{(n)} \in$ $L_{q}\left(\left[R_{1}, R_{2}\right]\right)$, for all $\omega \in S^{N-1}$. There exists $K_{3}>0$ such that $\left\|\left(f(s \omega) s^{N-1}\right)^{(n)}\right\|_{L_{q}\left(\left[R_{1}, R_{2}\right]\right)} \leq K_{3}$, where $s \in\left[R_{1}, R_{2}\right]$, for all $\omega \in S^{N-1}$.

Then

(i)

$$
\begin{aligned}
& \mid \int_{A} f(y) d y-\sum_{k=0}^{n-1} \frac{1}{(k+1) !}\left[\left(\int_{S^{N-1}}\left(f(s \omega) s^{N-1}\right)^{(k)}\left(R_{1}\right) d \omega\right)\left(t-R_{1}\right)^{k+1}\right. \\
+ & \left.(-1)^{k}\left(\int_{S^{N-1}}\left(f(s \omega) s^{N-1}\right)^{(k)}\left(R_{2}\right) d \omega\right)\left(R_{2}-t\right)^{k+1}\right] \mid \\
\leq & \frac{2 \pi^{\frac{N}{2}}}{\Gamma\left(\frac{N}{2}\right)} \frac{K_{3}}{(n-1) !\left(n+\frac{1}{p}\right)(p(n-1)+1)^{\frac{1}{p}}}\left[\left(t-R_{1}\right)^{n+\frac{1}{p}}+\left(R_{2}-t\right)^{n+\frac{1}{p}}\right],
\end{aligned}
$$

for all $t \in\left[R_{1}, R_{2}\right]$,

(ii) at $t=\frac{R_{1}+R_{2}}{2}$, the right hand side of (2.25) is minimized, and we get:

$$
\begin{aligned}
& \mid \int_{A} f(y) d y-\sum_{k=0}^{n-1} \frac{1}{(k+1) !} \frac{\left(R_{2}-R_{1}\right)^{k+1}}{2^{k+1}} \\
\times \quad & {\left[\int_{S^{N-1}}\left(f(s \omega) s^{N-1}\right)^{(k)}\left(R_{1}\right) d \omega+(-1)^{k} \int_{S^{N-1}}\left(f(s \omega) s^{N-1}\right)^{(k)}\left(R_{2}\right) d \omega\right] \mid } \\
\leq & \frac{\pi^{\frac{N}{2}}}{\Gamma\left(\frac{N}{2}\right)} \frac{K_{3}}{(n-1) !\left(n+\frac{1}{p}\right)(p(n-1)+1)^{\frac{1}{p}}} \frac{\left(R_{2}-R_{1}\right)^{n+\frac{1}{p}}}{2^{n-1-\frac{1}{q}}},
\end{aligned}
$$


(iii) if $\left(f(s \omega) s^{N-1}\right)^{(k)}\left(R_{1}\right)=\left(f(s \omega) s^{N-1}\right)^{(k)}\left(R_{2}\right)=0$, for all $\omega \in S^{N-1}$, (i.e. $\frac{\partial^{k}\left(f(s \omega) s^{N-1}\right)}{\partial s^{k}}$ vanish on $\partial B\left(0, R_{1}\right)$ and $\left.\partial B\left(0, R_{2}\right)\right)$ for all $k=0,1, \ldots, n-1$, we obtain

$$
\left|\int_{A} f(y) d y\right| \leq \frac{\pi^{\frac{N}{2}}}{\Gamma\left(\frac{N}{2}\right)} \frac{K_{3}}{(n-1) !\left(n+\frac{1}{p}\right)(p(n-1)+1)^{\frac{1}{p}}} \frac{\left(R_{2}-R_{1}\right)^{n+\frac{1}{p}}}{2^{n-1-\frac{1}{q}}},
$$

which is a sharp inequality,

(iv) more generally, for $j=0,1,2, \ldots, \bar{N} \in \mathbb{N}$, it holds

$$
\begin{aligned}
& \mid \int_{A} f(y) d y-\sum_{k=0}^{n-1} \frac{1}{(k+1) !}\left(\frac{R_{2}-R_{1}}{\bar{N}}\right)^{k+1}\left[j^{k+1}\left(\int_{S^{N-1}}\left(f(s \omega) s^{N-1}\right)^{(k)}\left(R_{1}\right) d \omega\right)\right. \\
& \left.+\quad(-1)^{k}(\bar{N}-j)^{k+1}\left(\int_{S^{N-1}}\left(f(s \omega) s^{N-1}\right)^{(k)}\left(R_{2}\right) d \omega\right)\right] \mid \\
& \leq \frac{2 \pi^{\frac{N}{2}}}{\Gamma\left(\frac{N}{2}\right)} \frac{K_{3}}{(n-1) !\left(n+\frac{1}{p}\right)(p(n-1)+1)^{\frac{1}{p}}}\left(\frac{R_{2}-R_{1}}{\bar{N}}\right)^{n+\frac{1}{p}}\left[j^{n+\frac{1}{p}}+(\bar{N}-j)^{n+\frac{1}{p}}\right],
\end{aligned}
$$

(v) if $\left(f(s \omega) s^{N-1}\right)^{(k)}\left(R_{1}\right)=\left(f(s \omega) s^{N-1}\right)^{(k)}\left(R_{2}\right)=0$, for all $\omega \in S^{N-1}$, (i.e. $\frac{\partial^{k}\left(f(s \omega) s^{N-1}\right)}{\partial s^{k}}$ vanish on $\partial B\left(0, R_{1}\right)$ and $\left.\partial B\left(0, R_{2}\right)\right)$ for $k=1, \ldots, n-1$, from (2.26) we get:

$$
\begin{aligned}
& \mid \int_{A} f(y) d y-\left(\frac{R_{2}-R_{1}}{\bar{N}}\right)\left[j R_{1}^{N-1}\left(\int_{S^{N-1}} f\left(R_{1} \omega\right) d \omega\right)\right. \\
+ & \left.(\bar{N}-j) R_{2}^{N-1}\left(\int_{S^{N-1}} f\left(R_{2} \omega\right) d \omega\right)\right] \mid \leq \frac{2 \pi^{\frac{N}{2}}}{\Gamma\left(\frac{N}{2}\right)} \\
\times & \frac{K_{3}}{(n-1) !\left(n+\frac{1}{p}\right)(p(n-1)+1)^{\frac{1}{p}}}\left(\frac{R_{2}-R_{1}}{\bar{N}}\right)^{n+\frac{1}{p}}\left[j^{n+\frac{1}{p}}+(\bar{N}-j)^{n+\frac{1}{p}}\right],
\end{aligned}
$$

for $j=0,1,2, \ldots, \bar{N} \in \mathbb{N}$,

(vi) when $\bar{N}=2$ and $j=1$, (2.27) turns to

$$
\begin{aligned}
& \left|\int_{A} f(y) d y-\left(\frac{R_{2}-R_{1}}{2}\right)\left(R_{1}^{N-1} \int_{S^{N-1}} f\left(R_{1} \omega\right) d \omega+R_{2}^{N-1} \int_{S^{N-1}} f\left(R_{2} \omega\right) d \omega\right)\right| \\
\leq & \frac{\pi^{\frac{N}{2}}}{\Gamma\left(\frac{N}{2}\right)} \frac{K_{3}}{(n-1) !\left(n+\frac{1}{p}\right)(p(n-1)+1)^{\frac{1}{p}}} \frac{\left(R_{2}-R_{1}\right)^{n+\frac{1}{p}}}{2^{n-1-\frac{1}{q}}},
\end{aligned}
$$

(vii) when $n=1$ (without any boundary conditions), we get from (2.28) that

$$
\begin{aligned}
& \left|\int_{A} f(y) d y-\left(\frac{R_{2}-R_{1}}{2}\right)\left(R_{1}^{N-1} \int_{S^{N-1}} f\left(R_{1} \omega\right) d \omega+R_{2}^{N-1} \int_{S^{N-1}} f\left(R_{2} \omega\right) d \omega\right)\right| \\
\leq & \frac{2^{\frac{1}{q}} \pi^{\frac{N}{2}} K_{3}}{\Gamma\left(\frac{N}{2}\right)\left(1+\frac{1}{p}\right)}\left(R_{2}-R_{1}\right)^{1+\frac{1}{p}} .
\end{aligned}
$$

Proof. Similar to the proof of Theorem 2.9. We apply Theorem 1.8 along with (1.16).

We continue with results on the ball. We present

Theorem 2.17. Consider $f: \overline{B(0, R)} \rightarrow \mathbb{R}$ be Lebesgue integrable, which is not necessarily radial. Assume that $f(s \omega) s^{N-1} \in A C([0, R])$, for all $\omega \in S^{N-1}, N \geq 2$. We further assume 
that $\frac{\partial f(s \omega) s^{N-1}}{\partial s} \in L_{\infty}([0, R])$, for all $\omega \in S^{N-1}$. Suppose there exists $M_{1}>0$ such that $\left\|\frac{\partial f(s \omega) s^{N-1}}{\partial s}\right\|_{\infty,(s \in[0, R])} \leq M_{1}$, for all $\omega \in S^{N-1}$.

Then

(i)

$$
\left|\int_{B(0, R)} f(y) d y-\left(\int_{S^{N-1}} f(R \omega) d \omega\right) R^{N-1}(R-t)\right| \leq \frac{\pi^{\frac{N}{2}} M_{1}}{\Gamma\left(\frac{N}{2}\right)}\left[t^{2}+(R-t)^{2}\right],
$$

for all $t \in[0, R]$,

(ii) at $t=\frac{R}{2}$, the right hand side of (2.29) is minimized, and we get:

$$
\left|\int_{B(0, R)} f(y) d y-\left(\int_{S^{N-1}} f(R \omega) d \omega\right) \frac{R^{N}}{2}\right| \leq \frac{\pi^{\frac{N}{2}} M_{1} R^{2}}{2 \Gamma\left(\frac{N}{2}\right)},
$$

(iii) if $f(R \omega)=0$, for all $\omega \in S^{N-1}$, (i.e. $f(\cdot \omega)$ vanishes on $\partial B(0, R)$ ), we obtain

$$
\left|\int_{B(0, R)} f(y) d y\right| \leq \frac{\pi^{\frac{N}{2}} M_{1} R^{2}}{2 \Gamma\left(\frac{N}{2}\right)}
$$

which is a sharp inequality,

(iv) more generally, for $j=0,1,2, \ldots, \bar{N} \in \mathbb{N}$, it holds

$$
\left|\int_{B(0, R)} f(y) d y-\frac{R^{N}}{\bar{N}}(\bar{N}-j) \int_{S^{N-1}} f(R \omega) d \omega\right| \leq \frac{\pi^{\frac{N}{2}} M_{1}}{\Gamma\left(\frac{N}{2}\right)}\left(\frac{R}{\bar{N}}\right)^{2}\left[j^{2}+(\bar{N}-j)^{2}\right],
$$

(v) when $\bar{N}=2$ and $j=1$, (2.30) turns to

$$
\left|\int_{B(0, R)} f(y) d y-\frac{R^{N}}{2} \int_{S^{N-1}} f(R \omega) d \omega\right| \leq \frac{\pi^{\frac{N}{2}} M_{1} R^{2}}{2 \Gamma\left(\frac{N}{2}\right)} .
$$

Proof. Same as the proof of Theorem 2.14, just set there $R_{1}=0$ and $R_{2}=R$ and use (1.15).

We continue with

Theorem 2.18. Consider $f: \overline{B(0, R)} \rightarrow \mathbb{R}$ be Lebesgue integrable, which is not necessarily radial. Assume that $f(s \omega) s^{N-1} \in A C([0, R])$, for all $\omega \in S^{N-1}, N \geq 2$. Suppose there exists $M_{2}>0$ such that $\left\|\frac{\partial f(s \omega) s^{N-1}}{\partial s}\right\|_{L_{1}([0, R])} \leq M_{2}$, for all $\omega \in S^{N-1}$.

Then

(i)

$$
\left|\int_{B(0, R)} f(y) d y-\left(\int_{S^{N-1}} f(R \omega) d \omega\right) R^{N-1}(R-t)\right| \leq \frac{2 \pi^{\frac{N}{2}} M_{2} R}{\Gamma\left(\frac{N}{2}\right)},
$$

for all $t \in[0, R]$,

(ii) if $f(R \omega)=0$, for all $\omega \in S^{N-1}$, (i.e. $f(\cdot \omega)$ vanishes on $\partial B(0, R)$ ) from (2.31), we obtain

$$
\left|\int_{B(0, R)} f(y) d y\right| \leq \frac{2 \pi^{\frac{N}{2}} M_{2} R}{\Gamma\left(\frac{N}{2}\right)}
$$

which is a sharp inequality, 
(iii) more generally, for $j=0,1,2, \ldots, \bar{N} \in \mathbb{N}$, it holds

$$
\left|\int_{B(0, R)} f(y) d y-\frac{R^{N}}{\bar{N}}(\bar{N}-j) \int_{S^{N-1}} f(R \omega) d \omega\right| \leq \frac{2 \pi^{\frac{N}{2}} M_{2} R}{\Gamma\left(\frac{N}{2}\right)},
$$

(iv) when $\bar{N}=2$ and $j=1$, (2.32) turns to

$$
\left|\int_{B(0, R)} f(y) d y-\frac{R^{N}}{2} \int_{S^{N-1}} f(R \omega) d \omega\right| \leq \frac{2 \pi^{\frac{N}{2}} M_{2} R}{\Gamma\left(\frac{N}{2}\right)} .
$$

Proof. Same as the proof of Theorem 2.15, just set there $R_{1}=0$ and $R_{2}=R$ and use (1.15).

We continue with

Theorem 2.19. Let $p, q>1: \frac{1}{p}+\frac{1}{q}=1$. Consider $f: \overline{B(0, R)} \rightarrow \mathbb{R}$ be Lebesgue integrable, which is not necessarily radial. Assume that $f(s \omega) s^{N-1} \in A C([0, R])$, for all $\omega \in S^{N-1}, N \geq 2$. We further assume that $\frac{\partial f(s \omega) s^{N-1}}{\partial s} \in L_{q}([0, R])$, for all $\omega \in S^{N-1}$. Suppose there exists $M_{3}>0$ such that $\left\|\frac{\partial f(s \omega) s^{N-1}}{\partial s}\right\|_{L_{q}([0, R])} \leq M_{3}$, for all $\omega \in S^{N-1}$.

Then

(i)

$$
\begin{aligned}
& \left|\int_{B(0, R)} f(y) d y-\left(\int_{S^{N-1}} f(R \omega) d \omega\right) R^{N-1}(R-t)\right| \\
\leq & \frac{2 \pi^{\frac{N}{2}} M_{3}}{\Gamma\left(\frac{N}{2}\right)\left(1+\frac{1}{p}\right)}\left[t^{1+\frac{1}{p}}+(R-t)^{1+\frac{1}{p}}\right],
\end{aligned}
$$

for all $t \in[0, R]$

(ii) at $t=\frac{R}{2}$, the right hand side of (2.33) is minimized, and we get:

$$
\left|\int_{B(0, R)} f(y) d y-\left(\int_{S^{N-1}} f(R \omega) d \omega\right) \frac{R^{N}}{2}\right| \leq \frac{2^{\frac{1}{q}} \pi^{\frac{N}{2}} M_{3} R^{1+\frac{1}{p}}}{\Gamma\left(\frac{N}{2}\right)},
$$

(iii) if $f(R \omega)=0$, for all $\omega \in S^{N-1}$, (i.e. $f(\cdot \omega)$ vanishes on $\partial B(0, R)$ ), we obtain

$$
\left|\int_{B(0, R)} f(y) d y\right| \leq \frac{2^{\frac{1}{q}} \pi^{\frac{N}{2}} M_{3} R^{1+\frac{1}{p}}}{\Gamma\left(\frac{N}{2}\right)}
$$

which is a sharp inequality,

(iv) more generally, for $j=0,1,2, \ldots, \bar{N} \in \mathbb{N}$, it holds

$$
\begin{aligned}
& \left|\int_{B(0, R)} f(y) d y-\frac{R^{N}}{\bar{N}}(\bar{N}-j) \int_{S^{N-1}} f(R \omega) d \omega\right| \\
\leq & \frac{2 \pi^{\frac{N}{2}} M_{3}}{\left(1+\frac{1}{p}\right) \Gamma\left(\frac{N}{2}\right)}\left(\frac{R}{\bar{N}}\right)^{1+\frac{1}{p}}\left[j^{1+\frac{1}{p}}+(\bar{N}-j)^{1+\frac{1}{p}}\right],
\end{aligned}
$$

(v) when $\bar{N}=2$ and $j=1$, (2.34) turns to

$$
\left|\int_{B(0, R)} f(y) d y-\frac{R^{N}}{2} \int_{S^{N-1}} f(R \omega) d \omega\right| \leq \frac{2^{\frac{1}{q}} \pi^{\frac{N}{2}} M_{3} R^{1+\frac{1}{p}}}{\left(1+\frac{1}{p}\right) \Gamma\left(\frac{N}{2}\right)} .
$$


Proof. Same as the proof of Theorem 2.16, just set there $R_{1}=0$ and $R_{2}=R$ and use (1.15).

\section{REFERENCES}

[1] R. P. Agarwal, S. S. Dragomir: An application of Hayashi's inequality for differentiable functions, Computers Math. Applic., 6 (1996), 95-99.

[2] G. A. Anastassiou: Fractional Differentiation Inequalities, Research Monograph, Springer, New York, 2009.

[3] G. A. Anastassiou: General Iyengar type inequalities, submitted, 2018.

[4] Xiao-Liang Cheng: The Iyengar-type inequality, Applied Math. Letters 14 (2001), 975-978.

[5] K. S. K. Iyengar: Note on an inequality, Math. Student 6, (1938), 75-76.

[6] Zheng Liu: Note on Iyengar's inequality, Univ. Beograd Publ. Elektrotechn. Fak., Ser. Mat. 16 (2005), $29-35$.

[7] F. Qi: Further generalizations of inequalities for an integral, Univ. Beograd Publ. Elektrotechn. Fak., Ser. Mat. 8 (1997), 79-83.

[8] W. Rudin: Real and Complex Analysis, International Student edition, Mc Graw Hill, London, New York, 1970.

[9] D. Stroock: A Concise Introduction to the Theory of Integration, Third Edition, Birkhaüser, Boston, Basel, Berlin, 1999.

DEPARTMENT OF MATHEMATICAL SCIENCES,

UNIVERSITY OF MEMPHIS,

MEMPHIS, TN 38152,

U.S.A.

E-mail address: ganastss@memphis.edu 